\title{
John Mallet-Paret 60
}

\author{
Shui-Nee Chow ${ }^{1}$ - Roger D. Nussbaum² . \\ Hans-Otto Walther ${ }^{3}$
}

Received: 31 August 2015 / Published online: 25 September 2015

C Springer Science+Business Media New York 2015

John (Joseph Douglas) Mallet-Paret was born on February 15, 1952, and grew up with three brothers in Edmonton, Alberta. John's father served in the Canadian Airforce in World War II and obtained a degree in Electrical Engineeering from the University of British Columbia after the war. John's mother was a homemaker.

From an early age John was fascinated by science. He went through several chemistry sets before the age of 10, and still describes with pleasure the eureka moment when he learned that a chemistry set was not just a toy and that chemistry could be an actual profession.

In his early teens, John received a telescope as a Christmas present; and for several years astronomy was a major preoccupation and pleasure, with frequent trips to the library to take out books on the subject. Gradually this led him in the direction of mathematics - "right ascension and declination" are really spherical coordinates - and he realized that the future positions of the planets could be predicted with the aid of tools like calculus.

John's father taught John some fundamentals of calculus and John learned a great deal on his own. This was a "course" with no proofs; but while John had no rigorous mathematics courses in high school, he understood that in Mathematics "if one did it right, there could be no dispute".

In 1968, John entered the University of Alberta in Edmonton. Jack Macki and others at the University had established an honors calculus sequence, and Macki had recruited many excellent students for this honors program. The textbook was Apostol's calculus book and the order of the day was mathematical rigor. John loved it, and it seems fair to say that a

\footnotetext{
$\bowtie$ Hans-Otto Walther

Hans-Otto.Walther@math.uni-giessen.de

Shui-Nee Chow

chow@math.gatech.edu

Roger D. Nussbaum

nussbaum@math.rutgers.edu

1 Georgia Institute of Technology, Atlanta, GA, USA

2 Rutgers University, Piscataway, NJ, USA

3 Universitaet Giessen, Giessen, Germany
} 
career in Mathematics was foreordained from that point on. It may be worth mentioning that this same honors program produced a number of other students who also went on to distinguished carreers in the academic world: Marcia Klawe is a computer scientist and President of Harvey Mudd College, Robert Skeel is is a Professor in the Department of Computer Science at Purdue University and Alfred Weiss is a Professor in the Mathematics Department at the University of Alberta.

In 1971 John graduated from the University of Alberta and, after some discussions with his Professors Jack Macki and Herb Freedman, decided to attend graduate school at the University of Minnesota. (Berkeley, Maryland and Warwick were other possibilities). At Minnesota, John gravitated in the direction of differential equations and dynamical systems. He took an advanced course on differential equations from Sibuya and a one semester topics course by McGehee which concentrated on the restricted three body problem and geometrical dynamical systems. This topics course later evolved into a working seminar which went through a number of (at that time) recent books: e.g., Hale's book on functional differential equations (sometimes called delay differential equations), Nitecki's book on dynamical systems, and the Abraham-Robbin book, Transversal Mappings and Flows.

Early on at Minnesota, John had thought that he might work with Ken Meyers as a Ph.D. advisor. However, Ken left Minnesota for Cincinnati for personal reasons. Later John met Larry Markus, and Markus and George Sell, were to become his joint Ph.D. advisors. Soon after their initial meeting, Markus told John that he really needed to attend an important conference on delay differential equations which would be held at Warwick. The requirement for an airplane ticket and expenses was that he take notes of all the talks. Other participants at the conference were A. Halanay, Jim Yorke, Jack Hale, Jake Levin, John Nohel, Mary Cartwright and George Sell; and it seems fair to say, in view of the later trajectory of John's work, that Markus's recommendation was, as the English say, "spot-on".

In the early 70's at Minnesota, there was a great deal of discussion of Smale's work. In his self-generated Ph.D. topic, John extended aspects of the Kupka-Smale theory to functional differential equations, which define dynamical systems on infinite-dimensional spaces. John's result required a thorough understanding of the most advanced recent work in finitedimensional dynamical systems and involved, among other matters, the invention of new perturbation techniques for functional differential equations [38]; see also [39,41]. The systematic exploration and presentation of functional differential equations from a dynamical systems point of view had been initiated only a few years earlier, by Jack Hale and others.

In 1974 John received his Ph.D. from the University of Minnesota. He gave a talk at the AMS meeting in San Francisco in January of that year describing his impressive Ph.D. results, and Jack Hale offered him a one year post-doc position at Brown for the following year. In the middle of 1975 , that one year offer was changed to a tenure track position. In 1978 John became associate professor. During the academic years 1981-1983 he took leave from Brown University and held a position at Michigan State University. In 1983 he accepted an offer to become a full professor at Brown University.

During these early years John began to work on a variety of problems in dynamical systems theory, both finite and, more often, infinite-dimensional, first by himself and then often jointly with Shui-Nee Chow, Jack Hale, and Jim Yorke. An outstanding result which John proved shortly after completing his Ph.D. thesis establishes that for continuously differentiable operators in Hilbert space, under appropriate additional conditions, compact negatively invariant sets (like global attractors, for example) are finite-dimensional [40]. Other remarkable results concern local and global bifurcations in one-parameter families of vector fields $[1,2,9,11,19-21,30,80]$, integral averaging and the Fuller index counting periodic orbits 
$[12,13]$, shells with small curvature [42], the obstacle problem [14,43,44], homology and homotopy methods of finding zeros [29,31,37].

With the exception of the work on the obstacle problem, the topics described above contribute to the general theory of dynamical systems. In the early 80's John began to study specific classes of nonlinear differential equations. A major part of his work since then has been devoted to autonomous delay differential equations. In this area already simple-looking scalar equations show a rich variety of dynamical phenomena, from stable periodic orbits to chaotic motion. John's first results on delay differential equations (after the thesis) appeared in 1983 [15,45]. 1986 is the year of John's first joint papers with Roger Nussbaum [54,55], on periodic solutions of a family of delay differential equations modeling the interaction of delayed negative and instantaneous feedback. They were followed by related papers $[56,58]$ in 1989 and 1993, and by work with S.-N. Chow and X. B. Lin [22]. The collaboration with Roger continues up to the present and resulted in a series of remarkable and beautiful results. We shall come back to some of them below. In 1988 John published an outstanding, seminal result about the global dynamics generated by a prototypical class of delay differential equations: A flow on a compact metric space which corresponds to the delay differential equation acting on its global attractor admits a Morse-Smale decomposition into level sets of a discrete Lyapunov functional, with heteroclinic connections between these level sets [46]. John's Lyapunov functional from [46] is a relative (if not precursor) of the lap number for solutions of scalar reaction-diffusion equations. It became a powerful tool for studying the long term behaviour of solutions to delay differential equations, and has been used by several authors. Related to [46] is work with Bernold Fiedler [32] which investigates the orbits connecting the level sets of the Morse-Smale decomposition.

In the same period of time, John also worked with colleagues on a variety of other, quite different problems involving ordinary and partial differential and integral equations, most of which were in some way or another related to applications. The topics include the Lyapunov dimension of a non-smooth attracting torus ([36], with J. L. Kaplan and J. A. Yorke), transition layers for semilinear boundary value problems ([3], with S. B. Angenent and L. Peletier), periodic solutions to Volterra integral equations ([18], with S.-N. Chow and O. Diekmann), bifurcation of travelling and standing waves on a circle ([79], with S. A. van Gils), and switching of optimal controls [6], with Pavol Brunovsky. Here is what Palo recalls about his collaboration with John: "Drinking coffee in the lounge of Michigan State during my 1980 stay, I was telling about an open problem on the finiteness of the number of switchings in control theory. I am not even sure I wrote down the 4th order equation on the blackboard. What I am sure, is that John immediately noticed a homogeneity of the equation which finally led to the solution of the problem. The paper (thanks to which I can be proud of having JMP number 1) appeared five years later. The story is symptomatic about John. I had the chance to work with him also later. While drawing pictures and discussing problems geometrically I could more or less cope with him. Yet, once we began to compute I became hopelessly lost. I could never understand how John knew to guess the appropriate power of scaling and I had the impression that in his mind he had the image of the formula, how it would look five steps later. Nevertheless he always remained patient and polite. The second part of the story is the length until publication of the result. With John, it was highly random, infinity having nonzero probability." Other collaborators have echoed Palo's remarks, the random length of time until publication being, in particular, a common lament.

Further topics from that period were chaos in a partial differential equation for a heated fluid ([81], with J. A. Yorke and E. D. Yorke, John's only paper in a physics journal), inertial manifolds ([72,73] with G. R. Sell, [77] with G. R. Sell and Z. Shao), Poincaré-Bendixson results for scalar reaction diffusion equations ([33], with B. Fiedler) and for monotone cyclic 
feedback systems of ordinary differential equations ([78], with H. L. Smith), the hypercycle system ([34], with J. Hofbauer and H. L. Smith), Josephson junctions ([4], with D. G. Aronson and M. Golubitsky), and Floquet theory for periodic parabolic partial differential equations $([23,24]$, with S.-N. Chow and K. Lu).

It must have been around the year 1990 that John Mallet-Paret and Roger Nussbaum began to extend their work on delay differential equations to the case of non-constant, statedependent delays. Historically, a few equations of this kind had been studied before, actually since the days of Poisson, and from time to time specific examples arose in physics, engineering, and in the life sciences. Compared to equations with constant delays differential equations with state-dependent delays can obviously provide more realistic models in applications. They are nonlinear by nature, and they are not covered by the familiar theory of retarded functional differential equations, due to a specific lack of smoothness. Before the turn of the millenium one did not even know how to linearize such equations. The work of Mallet-Paret and Nussbaum, since their first paper [57] on the subject from 1992 up to the present, is the first systematic study of the global dynamics of a prototypic family of differential equations with state-dependent delay. It revealed a fascinating collection of phenomena which show how a state-dependent delay shapes periodic solutions, forces uniqueness of their orbits, and provides so-called superstability of periodic orbits close to a singular limit $[59,62,67,68]$. Their paper [71] with John's student Panagiotis Paraskevopoulos is a rather general result on existence of periodic solutions. In this paper they also observed the smoothness property of being almost Fréchet differentiable, which in a slightly different version some years later turned out to be crucial in the search for suitable state spaces with differentiable solution operators on them, for equations with state-dependent delay (so that the missing linearization mystery was resolved, among other questions). The study of the profiles of periodic solutions in the singular limit led John and Roger to a new kind of topological eigenvalue problems which they studied in [60,61]. Ongoing research deals with the coexistence of analyticity and nonanalyticity in solutions to differential equations with variable delays [70].

The evolving theory of differential equations with variable delay owes another important stimulus to John, here in his role as supervisor. His student Hari Krishnan constructed the first local invariant manifold in this area, in his $\mathrm{Ph} . \mathrm{D}$. thesis of $1998^{1}$ (the missing linearization was circumvented by means of a known heuristic recipe which produced a linear equation from the given nonlinear equation).

Returning to constant delays, in 1996 there appeared two papers by John Mallet-Paret and George Sell on cyclic monotone feedback systems of delay differential equations, one with a fundamental a priori result on Floquet multipliers for linear periodic systems [74] and the other one [75] about planar dynamics of Poincaré-Bendixson type in $\omega$-limit sets of solutions to nonlinear autonomous systems. This must be seen in light of the fact that in contrast to studies of the global dynamics generated by a single delay differential equation there were almost no such results for systems of equations. In both papers an extension of John's discrete Lyapunov functional from [46] to the case of systems is instrumental.

A little earlier another area had begun to attract John's attention: These are infinite systems of ordinary differential equations indexed by a lattice, like $\mathbb{Z}^{2} \subset \mathbb{R}^{2}$, so-called lattice dynamical systems. Such systems arise in various applications. Challenging problems in this area are the existence of traveling waves, their speed, and stability properties. Complicated spatial patterns and then traveling waves in lattice dynamical systems became the main theme of John's publications of the years 1996-1999. He collaborated with several co-authors, notably

1 An analysis of singularly perturbed delay-differential equations and equations with state-dependent delays. Ph.D. thesis, Brown University, Providence (R. I.), 1998. See also H. P. Krishnan, Existence of unstable manifolds for a certain class of delay differential equations. Electronic J. Differ. Equ. 32 (2002), 1-13. 
S.-N. Chow [10,16,17], S.-N. Chow and E. Van Vleck [27,28], S.-N. Chow and W. Shen [26], J. W. Cahn and E. Van Vleck [7]. The first paper addressing traveling waves is [48], the latest one is [51]. Depending on the coupling in the lattice the traveling wave Ansatz yields a functional differential equation of mixed type for the waveform, with both delayed and advanced arguments. Such equations are different from the familiar neutral and retarded functional differential equations, and basic concepts of dynamical systems theory seem not appropriate for them. John established a Fredholm alternative type result for linear mixed type functional differential equations [49]. In collaboration with Sjoerd Verduyn Lunel he studied exponential dichotomies for them [53]. The papers [50,52] deal with global aspects of traveling waves and so-called crystallographic pinning where waves are at rest in certain directions and moving in directions nearby. In the much later paper [35] with Aaron Hoffman crystallographic pinning is studied as a universal phenomenon.

Aside from lattice dynamical systems and differential equations with state-dependent delays John continued to work on other topics, among them cyclic systems [47] and modeling reflex asymmetries [5], in a contribution with Fatihcan Atay to mathematical biology. With Shui-Nee Chow and Pavol Brunovsky he obtained Hartman-Grobman type results for singularly perturbed vector fields [8], worked with George Sell on time discretization of differential systems and Lyapunov functions [76], and in collaboration with Roger Nussbaum, on asymptotic fixed point theory [63], measures of noncompactness and generalizations of the Krein-Rutman theorem on spectra of cone maps [64-66]. The latter are related to their joint work on delay differential equations. In [69] they generalized John's work with George Sell on Floquet multipliers [74], by means of new tensor and exterior products of monodromy operators defined by periodic linear delay differential equations.

Many of John's papers are quite long. This reflects not only the nonlocal nature of the problems he studied, of course, but also that some of these problems opened new directions and required the laying of foundations. His style can be characterized by its use of elementary tools and techniques as far as possible, while keeping a formalistic framework and associated language to a minimum. It is interesting to observe this already in the first publications written in his early twenties. Often he was inspired by problems in nonlinear dynamics which were in the air at a time, and were related to an application, began work with collaborators and then continued to study the relevant mathematical structures in depth, in joint work or alone. Certainly Shui-Nee Chow had his influence, and later Roger Nussbaum, while traces of Jack Hale's perception and style also seem visible. The large number of prominent colleagues with whom John has worked and the variety of topics are impressive.

Much of John's collaborative work began during long visits. In the course of the years John took leave from his home university for extended research stays, at University of Warwick, Heriott-Watt University, Mathematisch Centrum Amsterdam, Institute of Mathematics and Applications at University of Minnesota, University of Maryland, Courant Institute, Michigan State University, Georgia Institute of Technology, the Lorentz Center of Leiden University, Rutgers University, and at other institutions in Slovakia, Germany, Canada, Taiwan, China, and in the United States. At Georgia Institute of Technology, at Leiden University and at the School of Mathematics of University of Minnesota he held visiting positions.

What has been said above about John's style of writing mathematics applies equally to his lectures, which are notable for presentations best suited to the particular audience. Beyond that they are remarkable for their clarity and focus on crucial points. It seems that John did not have to learn this; his lectures have always been characterized by these qualities. Not surprisingly, he has been a much sought after speaker at conferences. Recently he was nominated to be one of the Distinguished Speakers of the thematic programs at the Fields Institute. 
John has frequently been invited to give lecture series during his research stays. He taught at schools associated with the Mathematisch Centrum in Amsterdam, Bratislava, Marrakech, Taipei, Halifax (Canada), Cetraro (Italy), Santiago de Chile, Oviedo, Guangzhou, and Chengdu.

Visits and joint work reveal facets of a personality beyond scientific collaboration. John himself talks about a "simple and mathematical life"; his car is twenty years old and he has no television. As he recalls, Jack Hale and George Sell were role models for him, both with regard to mathematics and travelling; and similarly Joseph LaSalle was another influence, both in his mathematics and in his knowledge and appreciation of good food. John likes ethnic food, and knows very well how many of the fine specialities from all over the world are prepared (in theory, not being a cook himself). His friends and colleagues know of John's broad intellectual interests and appreciate his keen insight, in discussions about politics and in conversations about history, literature, movies, and more. Sports and exercise, however, are not favored topics of conversation.

The first graduate student of John, supervised jointly by him and Jack Hale, was Nicholas Alikakos who earned his Ph.D. in 1978 and now is a professor of mathematics at University of Athens. Nine others followed, among them Panagiotis Paraskevopoulos, Fatihcan Atay, Hari Krishnan, and Aaron Hoffman mentioned above.

Since 1998 John has served the community as an organizer of conferences, workshops and minisymposia, at Mathematisches Forschungsinstitut Oberwolfach (1998, with Roger Nussbaum and Hans-Otto Walther), at a SIAM National Meeting in Atlanta and a Dynamical Systems conference in Snowbird (1999), at Banff International Research Station (2004, with H. O. Walther and Jianhong Wu), at Fields Institute (2008, with J. Wu and Yingfei Yi), at Max Planck Institute for Physics of Complex Systems in Dresden (2009, with Jayme de Luca and Tony Humphries), and at American Institute of Mathematics in Palo Alto (2010, with R. D. Nussbaum and H. O. Walther).

Since 2000 John is Co-Editor-in-Chief of Journal of Differential Equations together with Shui-Nee Chow and Konstantin Mischaikow. He is also a member of the editorial board of Journal of Dynamics and Differential Equations as well as of Canadian Applied Mathematics Quarterly, Abstract and Applied Analysis, Dynamic Systems and Applications, Differential Equations and Dynamical Systems, Advanced Nonlinear Studies, Communications in Applied Analysis. Earlier he served on the editorial boards of Transactions of the American Mathematical Society and Discrete and Continuous Dynamical Systems-Series A.

At Brown University he was the Director of Lefschetz Center for Dynamical Systems (July 2002-June 2006, July 2007-June 2010) and served as Associate Chairman for the period July 2005-June 2006 and from July 2011 to the present.

\section{References}

1. Alligood, K.T., Mallet-Paret, J., Yorke, J.A.: Families of periodic orbits: local continuability does imply global continuability. J. Differ. Geom. 16, 483-492 (1981)

2. Alligood, K.T., Mallet-Paret, J., Yorke, J.A.: An index for the global continuation of relatively isolated sets of periodic orbits. In: Geometric Dynamics. Lecture Notes in Mathematics, vol. 1007, pp. 1-21. Springer, Heidelberg (1983)

3. Angenent, S.B., Mallet-Paret, J., Peletier, L.A.: Stable transition layers in a semilinear boundary value problem. J. Differ. Equ. 67, 212-242 (1987)

4. Aronson, D.G., Golubitsky, M., Mallet-Paret, J.: Ponies on a merry-go-round in large arrays of Josephson junctions. Nonlinearity 4, 903-910 (1991)

5. Atay, F.M., Mallet-Paret, J.: Modeling reflex asymmetries with implicit delay differential equations. Bull. Math. Biol. 60, 999-1015 (1998) 
6. Brunovsky, P., Mallet-Paret, J.: Switchings of optimal controls and the equation $y^{(4)}+|y|^{\alpha} \operatorname{sign} y=$ $0,0<\alpha<1$. Casopis pro Pestovani Matematiky 110, 302-313 (1985)

7. Cahn, J.W., Mallet-Paret, J., Van Vleck, E.: Traveling wave solutions for systems of ODE's on a twodimensional spatial lattice. SIAM J. Appl. Math. 59, 455-493 (1999)

8. Chow, S.-N., Brunovský, P., Mallet-Paret, J.: Theorems of Hartman-Grobman type for singuluarly perturbed vector fields (preprint, 2002)

9. Chow, S.-N., Mallet-Paret, J., Yorke, J.A.: A periodic orbit index which is a bifurcation invariant. In: Geometric Dynamics. Lecture Notes in Mathematics, vol. 1007, pp. 109-131. Springer, Heidelberg (1983)

10. Chow, S.-N., Mallet-Paret, J.: Recent progress in lattice dynamical systems. Jpn. Soc. Ind. Appl. Math., accepted for publication

11. Chow, S.-N., Mallet-Paret, J.: Hopf bifurcation and the method of averaging. In: Marsden, J., McCracken, M. (eds.) The Hopf Bifurcation. Springer, New York (1976)

12. Chow, S.-N., Mallet-Paret, J.: Integral averaging and bifurcation. J. Differ. Equ. 26, 112-159 (1977)

13. Chow, S.-N., Mallet-Paret, J.: The Fuller index and global bifurcation. J. Differ. Equ. 28, 66-85 (1978)

14. Chow, S.N., Mallet-Paret, J.: The parametrized obstacle problem. J. Nonlinear Anal. 4, 73-91 (1980)

15. Chow, S.-N., Mallet-Paret, J.: Singularly perturbed delay-differential equations. In: Chandra, J., Scott, A.C. (eds.) Coupled Nonlinear Oscillators, pp. 7-12. North Holland, Amsterdam (1983)

16. Chow, S.N., Mallet-Paret, J.: Pattern formation and spatial chaos in lattice dynamical systems I. IEEE Trans. Circuits Syst. 42, 746-751 (1995)

17. Chow, S.N., Mallet-Paret, J.: Pattern formation and spatial chaos in lattice dynamical systems II. IEEE Trans. Circuits Syst. 42, 752-756 (1995)

18. Chow, S.-N., Diekmann, O., Mallet-Paret, J.: Stability, multiplicity and global continuation of symmetric periodic solutions of a nonlinear Volterra integral equation. Jpn. J. Appl. Math. 2, 433-469 (1985)

19. Chow, S.-N., Hale, J.K., Mallet-Paret, J.: Applications of generic bifurcation I. Arch. Ration. Mech. Anal. 59, 159-188 (1975)

20. Chow, S.-N., Hale, J.K., Mallet-Paret, J.: Applications of generic bifurcation II. Arch. Ration. Mech. Anal. 62, 209-235 (1976)

21. Chow, S.-N., Hale, J.K., Mallet-Paret, J.: An example of bifurcation to homoclinic orbits. J. Differ. Equ. 37, 351-373 (1980)

22. Chow, S.-N., Lin, X.-B., Mallet-Paret, J.: Transition layers for singularly perturbed delay differential equations with monotone nonlinearities. J. Dyn. Differ. Equ. 1, 3-43 (1989)

23. Chow, S.-N., Lu, K., Mallet-Paret, J.: Floquet theory for parabolic differential equations. J. Differ. Equ. 109, 147-200 (1994)

24. Chow, S.-N., Lu, K., Mallet-Paret, J.: Floquet bundles for scalar parabolic equations. Arch. Ration. Mech. Anal. 129, 245-304 (1995)

25. Chow, S.N., Mallet-Paret, J.: Obituary of Jack K. Hale. J. Dyn. Differ. Equ. 22, 73-78 (2010)

26. Chow, S.-N., Mallet-Paret, J., Shen, W.: Traveling waves in lattice dynamical systems. J. Differ. Equ. 149, 248-291 (1998)

27. Chow, S.-N., Mallet-Paret, J., Van Vleck, E.: Dynamics of lattice differential equations. Int. J. Bifurc. Chaos 6, 1605-1621 (1996)

28. Chow, S.-N., Mallet-Paret, J., Van Vleck, E.: Pattern formation and spatial chaos in spatially discrete evolution equations. Random Comput. Dyn. 4, 109-178 (1996)

29. Chow, S.-N., Mallet-Paret, J., Yorke, J.A.: Finding zeros of maps: homology methods that are constructive with probability one. Math. Comput. 32, 887-899 (1978)

30. Chow, S.-N., Mallet-Paret, J., Yorke, J.A.: Global Hopf bifurcation from a multiple eigenvalue. J. Nonlinear Anal. 2, 753-763 (1978)

31. Chow, S.-N., Mallet-Paret, J., Yorke, J.A.: A homotopy method for locating all zeros of a system of polynominals. In: Peitgen, H.O., Walther, H.O. (eds.) Functional Differential Equations and Approximation of Fixed Points. Lecture Notes in Mathematics, vol. 730, pp. 77-88. Springer, Heidelberg (1979)

32. Fiedler, B., Mallet-Paret, J.: Connections between Morse sets for delay-differential equations. J. Reine Angew. Math. 397, 23-41 (1989)

33. Fiedler, B., Mallet-Paret, J.: The Poincare-Bendixson theorem for scalar reaction diffusion equations. Arch. Ration. Mech. Anal. 107, 325-345 (1989)

34. Hofbauer, J., Mallet-Paret, J., Smith, H.L.: Stable periodic solutions for the hypercycle system. J. Dyn. Differ. Equ. 3, 423-436 (1991)

35. Hoffman, A., Mallet-Paret, J.: Universality of crystallographic pinning. J. Dyn. Differ. Equ. 22, 79-119 (2010)

36. Kaplan, J.A., Mallet-Paret, J., Yorke, J.A.: The Lyapunov dimension of a nowhere differentiable attracting torus. Ergod. Theory Dyn. Syst. 4, 261-281 (1984) 
37. Li, T.Y., Mallet-Paret, J., Yorke, J.A.: Regularity results for real analytic homotopies. Numer. Math. 46, 43-50 (1985)

38. Mallet-Paret, J.: Generic properties of fixed points and periodic orbits of retarded functional differential equations. Ph. D. thesis, Minneapolis, (1974)

39. Mallet-Paret, J.: Generic properties of retarded functional differential equations. Bull. Am. Math. Soc. 81, 750-752 (1975)

40. Mallet-Paret, J.: Negatively invariant sets of compact maps and an extension of a theorem of Cartwright. J. Differ. Equ. 22, 331-348 (1976)

41. Mallet-Paret, J.: Generic periodic solutions of functional differential equations. J. Differ. Equ. 25, 163-183 (1977)

42. Mallet-Paret, J.: Buckling of cylindrical shells with small curvature. Q. Appl. Math. 35, 383-400 (1977)

43. Mallet-Paret, J.: Generic unfoldings and normal forms of some singularities arising in the obstacle problem. Duke Math. J. 46, 645-683 (1979)

44. Mallet-Paret, J.: Generic bifurcation in the obstacle problem. Q. Appl. Math. 37, 355-387 (1980)

45. Mallet-Paret, J.: Morse decompositions and global continuation of periodic solutions of singularly perturbed delay equations. In: Ball, J.M. (ed.) Systems of Nonlinear Partial Differential Equations, pp. 351-365. D. Reidel, Dordrecht (1983)

46. Mallet-Paret, J.: Morse decompositions for delay-differential equations. J. Differ. Equ. 72, 270-315 (1988)

47. Mallet-Paret, J.: Stability and oscillation in nonlinear cyclic systems. In: Martelli, M., Cooke, K., Cumberbatch, E., Tang, B., Thieme, H. (eds.) Differential Equations and Applications to Biology and Industry, pp. 337-346. World Scientific, Singapore (1996)

48. Mallet-Paret, J.: Spatial patterns, spatial chaos, and traveling waves in lattice differential equations. In: van Strien, S.J., Verduyn Lunel, S.M. (eds.) Stochastic and Spatial Structures of Dynamical Systems, pp. 105-129. North-Holland, Amsterdam (1996)

49. Mallet-Paret, J.: The Fredholm alternative for functional differential equations of mixed type. J. Dyn. Differ. Equ. 11, 1-47 (1999)

50. Mallet-Paret, J.: The global structure of traveling waves in spatially discrete systems. J. Dyn. Differ. Equ. 11, 49-127 (1999)

51. Mallet-Paret, J.: Traveling waves in spatially discrete systems. In: Macki, J.W., Zecca, P. (eds.) Dynamical Systems. Lecture Notes in Mathematics, vol. 1822, pp. 231-298. Springer, Heidelberg (2003)

52. Mallet-Paret, J.: Crystallographic pinning: direction dependent pinning in lattice differential equations (preprint, 2001)

53. Mallet-Paret, J., Verduyn Lunel, S.: Exponential dichotomies and Wiener-Hopf factorization for mixedtype functional differential equations via holomorphic factorization (preprint, 2000)

54. Mallet-Paret, J., Nussbaum, R.D.: Global continuation and asymptotic behavior for periodic solutions of a differential-delay equation. Ann. Mat. Pura Appl. 145, 33-128 (1986)

55. Mallet-Paret, J., Nussbaum, R.D.: A bifurcation gap for a singularly perturbed delay equation. In: Barnsley, M.F., Demko, S.G. (eds.) Chaotic Dynamics and Fractals, pp. 263-286. Academic Press, Waltham (1986)

56. Mallet-Paret, J., Nussbaum, R.D.: A differential-delay equation arising in optics and physiology. SIAM J. Math. Anal. 20, 249-292 (1989)

57. Mallet-Paret, J., Nussbaum, R.D.: Boundary layer phenomena for differential delay equations with state dependent time lags I. Arch. Ration. Mech. Anal. 120, 99-146 (1992)

58. Mallet-Paret, J., Nussbaum, R.D.: Multiple transitions layers in a singularly perturbed differential-delay equation. Proc. R. Soc. Edinb. 123A, 1119-1134 (1993)

59. Mallet-Paret, J., Nussbaum, R.D.: Boundary layer phenomena for differential delay equations with state dependent time lags II. J. Reine Angew. Math. 477, 129-197 (1996)

60. Mallet-Paret, J., Nussbaum, R.D.: Eigenvalues for a class of homogeneous cone maps arising from maxplus operators. Discrete Cont. Dyn. Syst 8, 519-562 (2002)

61. Mallet-Paret, J., Nussbaum, R.D.: A basis theorem for a class of max-plus eigenproblems. J. Differ. Equ. 189, 616-639 (2003)

62. Mallet-Paret, J., Nussbaum, R.D.: Boundary layer phenomena for delay-differential equations with state dependent time lags III. J. Differ. Equ. 189, 640-692 (2003)

63. Mallet-Paret, J., Nussbaum, R.D.: Asymptotic fixed point theory and the beer barrel theorem. J. Fixed Point Theory Appl. 4, 203-245 (2008)

64. Mallet-Paret, J., Nussbaum, R.D.: Generalizing the Krein-Rutman theorem, measures of noncompactness and the fixed point index. J. Fixed Point Theory Appl. 7, 103-143 (2010)

65. Mallet-Paret, J., Nussbaum, R.D.: Inequivalent measures of noncompactness and the radius of the essential spectrum. Proc. Am. Math. Soc. 139, 917-930 (2011)

66. Mallet-Paret, J., Nussbaum, R.D.: Inequivalent measures of noncompactness. Annali di Matematica 190, 453-488 (2011) 
67. Mallet-Paret, J., Nussbaum, R.D.: Superstability and rigorous asymptotics in singularly perturbed statedependent delay differential equations. J. Differ. Equ. 250, 4037-4084 (2011)

68. Mallet-Paret, J., Nussbaum, R.D.: Stability of periodic solutions of state-dependent delay differential equations. J. Differ. Equ. 250, 4085-4103 (2011)

69. Mallet-Paret, J., Nussbaum, R.D.: Tensor products, positive linear operators and delay-differential equations. J. Dyn. Differ. Equ. 25, 843-905 (2013)

70. Mallet-Paret, J., Nussbaum, R.D.: Analyticity and nonanalyticity of solutions of delay-differential equations. SIAM J. Math. Anal. 46, 2468-2500 (2014)

71. Mallet-Paret, J., Nussbaum, R.D., Paraskevopoulos, P.: Periodic solutions for functional differential equations with multiple state-dependent time lags. Topol. Methods Nonlinear Anal. 3, 101-162 (1994)

72. Mallet-Paret, J., Sell, G.R.: The principle of spatial averaging and inertial manifolds for reaction-diffusion equations. In: Nonlinear Semigroups, Partial Differential Equations and Attractors. Lecture Notes in Mathematics, vol. 1248, pp. 94-107. Springer, Heidelberg (1987)

73. Mallet-Paret, J., Sell, G.R.: Inertial manifolds for reaction-diffusion equations in higher space dimensions. J. Am. Math. Soc. 1, 805-866 (1988)

74. Mallet-Paret, J., Sell, G.R.: Systems of differential delay equations: Floquet multipliers and discrete Lyapnov functions. J. Differ. Equ. 125, 385-440 (1996)

75. Mallet-Paret, J., Sell, G.R.: The Poincaré-Bendixson theorem for monotone cyclic feedback systems with delay. J. Differ. Equ. 125, 441-489 (1996)

76. Mallet-Paret, J., Sell, G.R.: Differential systems with feedback: time discretization and Lyapunov functions. Discrete Cont. Dyn. Syst. 15, 659-698 (2003)

77. Mallet-Paret, J., Sell, G.R., Shao, Z.: Obstructions to the existence of normally hyperbolic inertial manifolds. Indiana Univ. Math. J. 42, 1027-1055 (1993)

78. Mallet-Paret, J., Smith, H.L.: The Poincare-Bendixson theorem for monotone cyclic feedback systems. J. Dyn. Differ. Equ. 2, 367-421 (1990)

79. Mallet-Paret, J., van Gils, S.: Hopf bifurcation and symmetry: traveling and standing waves on the circle. Proc. R. Soc. Edinb. 104A, 279-307 (1986)

80. Mallet-Paret, J., Yorke, J.A.: Snakes: oriented families of periodic orbits, their sources, sinks and continuation. J. Differ. Equ. 43, 419-450 (1982)

81. Mallet-Paret, J., Yorke, J.A., Yorke, E.D.: Lorenz-like chaos in a partial differential equation for a heated fluid loop. Phys. D 24, 279-291 (1987) 